\title{
A. Antoine, et D. Marguerie (dir.), 2007 - Bocages et Sociétés
}

\section{Vincent Carpentier}

\section{Q OpenEdition}

\section{Journals}

Édition électronique

URL : http://journals.openedition.org/rao/751

DOI : 10.4000/rao.751

ISBN : 978-2-7535-1608-3

ISSN : $1775-3732$

\section{Éditeur}

Presses universitaires de Rennes

\section{Édition imprimée}

Date de publication : 20 décembre 2008

Pagination : 282-288

ISBN : 978-2-7535-0789-0

ISSN : 0767-709X

\section{Référence électronique}

Vincent Carpentier, «A. Antoine, et D. Marguerie (dir.), 2007 - Bocages et Sociétés », Revue

archéologique de l'Ouest [En ligne], 25 | 2008, mis en ligne le 20 décembre 2008, consulté le 03

décembre 2020. URL : http://journals.openedition.org/rao/751 ; DOI : https://doi.org/10.4000/rao.751

Tous droits réservés 
un chapitre complet, les auteurs mettent l'accent sur une alimentation associant produits de l'élevage, céréales, légumes verts et fruits. Cette partie de l'ouvrage est particulièrement importante puisque les données exposées ici sont les premières à faire état d'une agriculture régionalement bien développée avant la Conquête romaine, alors que les sources écrites, et en particulier Strabon (Géographie, Livre III), dressaient un portrait des asturiens assez dévalorisant : population inculte et de nature sauvage, se nourrissant de glands, de châtaignes et de viande de chèvre!

Les deux derniers chapitres de l'ouvrage sont de portée plus générale et n'apportent que peu d'éléments pertinents à propos de la mise en perspective de cette découverte. Ils font un point sur la métallurgie au Bronze final à partir de l'analyse des deux bracelets découverts aux poignets de la femme de Fuentenegroso puis une présentation des gravures rupestres du Néolithique et de l'âge du Bronze de Peña Tú. La publication se solde par une bibliographie sommaire, mais sans une conclusion qui aurait été pourtant d'un grand intérêt pour revenir sur toutes les données acquises grâce à l'étude de ce site.
Malgré ces dernières remarques, on ne peut que saluer la qualité de la (re)construction archéologique faite autour de la femme de Fuentenegroso. Les textes sont concis et à la portée de tous. Les images sont de très bonne qualité et toujours informatives. Les analyses du contexte archéologique et les présentations plus générales visent à nous faire revivre la vie dans cette partie des Asturies il y a presque 3000 ans et il faut reconnaître que les auteurs y arrivent malgré la faiblesse de la documentation. Ils nous font alors découvrir par le prisme de cette inhumation le quotidien de cette femme, loin des préjugés issus des écris antiques. En bref, cet ouvrage est un bon exemple de ce qu'il est possible de produire, pour le public spécialiste ou non, pour peu que le travail soit mené de façon interdisciplinaire et méthodique, autour d'une découverte qui, initialement, pouvait paraittre assez «banale ».

Cyril MarcignY

(INRAP Basse-Normandie et UMR6566 - CReAAH)

Antoine, A. et Marguerie, D. (dir.), 2007 - Bocages et Sociétés, Actes du colloque organisé àl'université de Rennes 2 (29 septembre- $^{\text {er }}$ octobre 2004) par le CERHO (FRE 3004 - Centre de Recherche historique de l'Ouest) et le CREAAH (UMR 6566 - Centre de Recherche en Archéologie, Archéosciences et Histoire), Rennes, Presses universitaires de Rennes, coll. «Espaces et Territoires », 509 p., 24 pl. Couleurs en insert (ISBN 978-2-7535-0454-7; $23 €$ ).

Ces actes de colloque dédiés à l'histoire du bocage dans tous ses états composent un fort volume de quelque cinq cents pages, conçu comme une réactualisation depuis la table ronde "Les bocages : histoire, écologie, économie. Aspects physiques, biologiques et humains des écosystèmes bocagers des régions tempérées humides » organisée à Rennes par le CNRS, l'INRA et l'ENSAA en 1976. Selon la formule consacrée des " trente ans après ", l'ouvrage codirigé par Annie Antoine et Dominique Marguerie, respectivement historienne à l'Université de Rennes 2 et paléoécologue du CNRS, affirme une orientation résolument interdisciplinaire des recherches portant sur les systèmes bocagers, en particulier ceux de l'Ouest français, depuis les avancées fondatrices d'Henri Sée pour la Bretagne ou René Musset pour le Bas-Maine. Pour ces icônes de la géographie bocagère $\mathrm{du} \mathrm{xx}^{\mathrm{e}}$ siècle commençant, le bocage se concevait en effet comme un type ou une forme spécifique d'organisation de l'espace rural à laquelle s'est intéressée par la suite, à partir de l'après-Guerre, une nouvelle génération de géographes, à l'image d'André Meynier puis Pierre Flatrès. Influencés par le structuralisme, ces chercheurs de l' " école de Rennes » se sont focalisés sur le concept global de " paysage agraire " et l'idée d'un " arc atlantique paysager ", coïncidant avec une " unité de civilisation » identifiable de la Galice au Devon et à la Norvège sur la base d'une communauté d'usages et de paysages. Ces mêmes années 1950-1970 furent le cadre d'une distanciation générale des historiens visà-vis de cette dimension paysagère, considérée comme l'apanage des géographes en dépit pourtant du caractère central qu'elle revêt au sein d'une histoire rurale qui, dans les faits, restait essentiellement envisagée sous ses aspects sociaux et économiques, plus rarement techniques, aux dépens de ses réalités agraires et environnementales. Malgré plusieurs interpellations, émanant d'ailleurs de géographes, en faveur d'une prise en considération accrue des phénomènes de mobilitél résilience des paysages et en particulier du bocage au cours des quatre ou cinq derniers siècles, le renouveau méthodo- et épistémologique de l'histoire rurale n'est véritablement opéré que dans les années quatre-vingt-dix, à l'aune des travaux consacrés par Annie Antoine au " paysage de l'historien " et à une " archéologie des bocages de l'ouest de la France " héritière du concept aujourd'hui fort discuté d'" archéologie du paysage ", qui ont jeté les bases concrètes d'une véritable passerelle interdisciplinaire entre l'histoire et la géographie. Dans le même temps, les disciplines naturalistes se sont penchées 
elles aussi sur le thème du bocage, dénonçant le mythe fixiste $\mathrm{du}$ " milieu naturel en équilibre » au profit d'une large ouverture en direction des sources historiques et archéologiques - celles-ci de plus en plus étoffées grâce à l'essor de l'archéologie préventive - propres à retracer les étapes successives de l'évolution des systèmes paysagers à travers la mise en exergue d'un ensemble d'héritages biologiques, techniques, socio-économiques interagissant dans l'histoire du couple sociétésmilieux. Ainsi qu'en témoignent les travaux de Dominique Marguerie sur l'histoire végétale de l'Ouest armoricain, ces dimensions biologiques et physiques, spatiales, techniques et anthropologiques du paysage s'interpénètrent désormais dans cette nouvelle conception heuristique et multiscalaire, désormais inscrite dans la longue durée. Fort de ces évolutions, le colloque "Bocages et Sociétés " tenu à Rennes en 2004 est le fruit d'un travail collectif et interdisciplinaire engagé par un groupe de vingt-deux chercheurs autour d'un projet constitué en réponse à l'appel d'offres « Histoire des interactions sociétés-milieux " (HISM), financé dans le cadre du programme «Environnement-Vie-Sociétés » du CNRS, de 1999 à 2001. La question centrale de ce projet est celle des interactions entre phénomènes sociétaux et évolution de l'environnement. Elle se décline notamment à travers deux thèmes privilégiés qui sont celui de l'évolution sur le temps long du couple société/paysage, et celui de l'impact des pratiques sociales actuelles sur les structures bocagères anciennes. La méthodologie mise en œuvre s'inscrit à la croisée des disciplines archéologique, historique, paléoécologique et écologique, géographique et agronomique. Les nécessaires rencontres entre chercheurs ont notamment été favorisées par le recrutement de deux sites-ateliers de nature complémentaire, tous deux situés en Ille-et-Vilaine : celui de PleineFougères, étudié par les écologues depuis 1993, et celui de Montours, sur lequel ont été mis au jour d'importants vestiges d'établissements ruraux remontant pour l'essentiel au haut Moyen Âge (fouilles d'Isabelle Catteddu, Inrap). Cette publication vient à la suite d'une première communication de synthèse parue à l'occasion du bilan de l'appel d'offres HISM "Des milieux et des hommes : fragments d'histoire croisée ", sous la direction de T. Muxart, F.-D. Vivien, B. Villaba et J. Burnouf en 2003. Elle présente aujourd'hui une somme de trente-cinq communications respectivement consacrées à l'historiographie et la thématique générale du colloque (2 communications), ainsi qu'à quatre thèmes davantage spécifiques, déclinés au fil de quelque quatre cents pages : thème $1:$ «Recherche des origines et suivi des interactions sociétés/ paysages sur le temps long" (7 communications); thème 2 : "Des structures et des fonctions évolutives » (10); thème 3 : "Les sociétés bocagères " (10); thème $4:$ : L'évolution des idées sur le bocage » (6). Suivent en fin de volume les résumés bilingues (français-anglais) ainsi que la liste des auteurs.
En ouverture à ces actes, J. Baudry et $S$. Périchon nous livrent un exposé général sur la littérature internationale consacrée aux aspects structurels et fonctionnels du couple haie-bocage (p. 22-32). Il apparaît que les dimensions sociales des réseaux de haies affichent une indéniable variété et peuvent en outre connaître une évolution relativement rapide selon les lieux et les époques, tandis que les réseaux bocagers proprement dits, considérés à une échelle plus étendue, se montrent au contraire plutôt stables dans la durée. Les exemples déclinés à l'appui de cet exposé démontrent le potentiel particulier qu'offre cet objet de recherche pour une rencontre entre sciences naturelles et sociales, en liaison notamment avec la dimension composite, environnementale, anthropologique et technique, des haies et du bocage, ainsi qu'avec la co-évolution de ces facteurs au fil du temps (usages fourragers, limites parcellaires et clôtures, production de bois, rôle de régulation hydroclimatique, rempart contre l'érosion des sols, drainage, filtre contre la pollution, maintien de la biodiversité, esthétique paysagère...). Dans la communication suivante, P. Mérot et F. Bridet-Guillaume proposent une bibliographie critique des recherches dédiées aux bocages depuis les années 1960 (p. 33-48). Ils mettent l'accent sur l'explosion de la littérature bocagère depuis les années 1990, en rapport avec un renversement des perspectives qui fut à l'origine de trois tendances actuellement dominantes, fondées sur : l'utilité environnementale objective des bocages en tant que facteurs de résilience des systèmes biophysiques; la fonction subjective du paysage bocager dans le champ de l'histoire, du patrimoine et des perceptions, notamment symboliques; l'utilité sociale reconnue au bocage dans les pratiques d'aménagement, ce troisième axe incluant les deux précédents. Ce triptyque s'inscrit dans un contexte de revalorisation des bocages qui succède à leur dépréciation globale au cours des années 1970-1980, visant dorénavant à réinsérer le bocage ou " néo-bocage ", en référence au système de production agrotechnique aujourd'hui dépassé dont il est le produit, dans un contexte socio-paysager au sein duquel il occupe une place centrale. L'analyse de la bibliographie proposée par les auteurs met en exergue les lacunes de la recherche concernant les bocages, et notamment celles relatives à la place de l'élevage.

Les communications réunies dans le thème 1 relèvent d'approches paléoenvironnementales, historiques et archéologiques croisées, fruit d'une orientation interdisciplinaire encore récente des recherches portant sur l'histoire bocagère de l'Ouest français. Les principaux objets concernés par cette recherche sont les réseaux parcellaires, leurs origines et leur évolution jusqu'au cadastre ancien; les formes végétales et notamment la haie au cours du temps; les formes d'établissements insérés dans les systèmes bocagers de l'Âge du Fer au Moyen Âge finissant. Sont également abordées la question de 
la place du bocage dans la longue durée de l'évolution paysagère, de même que celle des modalités présidant à la fabrication du bocage au début de l'ère contemporaine. Échelles de temps et d'espace sont utilisées de façon différente en réponse à la nature diverse des sources mises à contribution. Les données paléoenvironnementales, à savoir paléobotaniques et paléosédimentaires, permettent de retracer l'histoire de la végétation et des sols au cours des dix derniers millénaires, dans un contexte de plus en plus dominé par la main de l'homme. La communication que nous livrent A. Gebhardt, D. Marguerie, V. Bernard et L. Gaudin invite à discerner dans cette histoire végétale et sédimentaire trois temps forts pour l'Ouest armoricain (p. 51-61). Une première évolution liée à la pression anthropique atteint la chênaie diversifiée dense à tilleuls et ormes qui caractérise l'optimum climatique de l'Atlantique, vers 8500 avant notre ère, sur les côtes sudbretonnes. Ces ouvertures pionnières deviennent très nettes à compter de -7000 , précédant d'un millénaire l'expansion des mégalithes sur le pourtour du Golfe du Morbihan. D’une manière générale, le Néolithique est illustré par un impact anthropique beaucoup plus marqué sur les côtes que dans l'intérieur de la Bretagne. Un deuxième temps fort prend effet avec le Second Âge du Fer, environ quatre siècles avant notre ère, avec l'émergence d'un paysage agricole au sein duquel les cultures l'emportent sur le couvert forestier. Cette phase voit la généralisation des réseaux parcellaires et l'accentuation notable de l'érosion pédologique. L'époque gallo-romaine s'inscrit dans le prolongement de cette tendance, avant qu'un essoufflement s'amorce au cours du BasEmpire. Un troisième temps fort prend place par la suite au cours du haut Moyen Âge, et aboutit dès les $\mathrm{IX}^{\mathrm{e}}-\mathrm{X}^{\mathrm{e}}$ siècles à l'émergence d'un paysage agraire caractéristique de l'espace armoricain tel qu'il se définit de nos jours. À la suite d'une évolution encore mal connue, les premiers indices paléoenvironnementaux traduisant l'existence d'un véritable processus d'embocagement, fondé sur le développement de la haie de chênes, se manifestent dans les enregistrements polliniques vers la fin du XIv et au cours du siècle suivant.

Ce canevas forme le cadre général au sein duquel s'inscrivent les communications relatives à l'Antiquité romaine (P. Naas, p. 63-80), à l'époque carolingienne (I. Catteddu, p. 81-90), ou au bas Moyen Âge (J.-C. Meuret, p. 91-104). Des détails sont également fournis concernant l'étude palynologique des haies bocagères du Nord-Ouest de la France (D. Marguerie et J.-C. Oillic, p. 105-119), ou la lecture anthropologique des réseaux bocagers confrontée aux données de l'archéologie et de l'histoire, en particulier médiévales (M. Watteaux, p. 121-131). Une dernière contribution traite du coût de création d'un bocage productif au XIX siècle (R. Bourrigaud, p. 133-143). Les préoccupations des auteurs sont ici très imbriquées mais offrent des regards contrastés sur la question du bocage et de ses origines. La contribution de P. Naas concerne en réalité « l'avant bocage » des premiers siècles de notre ère, à la lumière de la prospection aérienne et de l'archéologie préventive. Cet antécédent paysager se traduit par un corpus d'enclos associés à des microparcellaires antérieurs au réseau de haies bocagères moderne. Tandis que les parcellaires associés aux villae s'avèrent encore globalement mal renseignés, il existe également des réseaux relativement vastes qui paraissent indépendants des habitats antiques et dont la fonction de même que la signification socio-économique restent à étudier. Le cas du site altomédiéval de Montours, présenté par I. Catteddu, constitue quant à lui un intéressant contre-exemple de continuité ou du moins, de rémanence formelle, entre l'avant bocage et le bocage moderne. On constate cependant, à travers le panel de données paléoenvironnementales associées à cet habitat du haut Moyen Âge, la rupture très nette qui coïncide avec l'époque mérovingienne vis-à-vis de l'environnement végétal des occupations antérieures. Le haut Moyen Âge se traduit ici par une puissante ouverture du milieu, concomitante de l'essor des activités agropastorales dans un fond de vallée jusqu'alors peu anthropisé. Puis, une nouvelle phase de déprise accompagne l'abandon de ce village au $\mathrm{x}^{\mathrm{e}}$ siècle et s'étale jusqu'à la fin du Moyen Âge, tandis que la structure parcellaire continue d'évoluer jusqu'à l'ère contemporaine, aboutissant à la mise en place de la géométrie bocagère actuelle. Du reste, ces données ne remettent pas en cause le fait aujourd'hui généralement admis que l'acte de naissance du bocage breton semble bel et bien se situer à la toute fin du Moyen Âge, entre le $\mathrm{Xv}^{\mathrm{e}}$ et le début $\mathrm{du} \mathrm{XVI}^{\mathrm{e}}$ siècle. C'est en tout cas ce qu'illustrent avec une appréciable précision l'exemple du parcellaire associé au manoir médiéval et moderne de la Montagne de Visseiche, présenté par J.-C. Meuret, de même que les données palynologiques recueillies par D. Marguerie et J.-C. Oillic en Haute-Bretagne.

Sur la base de ces données relatives au passé du bocage, M. Watteaux propose une relecture archéogéographique de la problématique des origines. Elle rappelle ainsi que dans $90 \%$ des cas recensés en Bretagne, les occupations pré-modernes accusent une nette discordance au regard de la trame bocagère contemporaine. L'accent est mis ensuite sur les problèmes d'échelle et de vocabulaire suscités par la réflexion actuelle sur les bocages de l'Ouest. Passant à une échelle de lecture globale beaucoup plus étendue que celle du site ou de l'anomalie étudiés par l'archéologue, M. Watteaux met en évidence des situations de concordance géométrique entre formes anciennes et modernes, y compris au sein des régions bocagères, qu'elle replace dans un contexte évolutif de longue durée. À partir de ces constatations se fait jour la nécessité d'une "reformulation de la question du bocage dans la lecture historique des paysages agraires français » 
(p. 129), à l'encontre de la thèse identitaire développée par l'historiographie qui aurait conduit à une surdétermination de l'objet bocage au sein de la réflexion historique. Dans la dernière communication de ce thème $1, \mathrm{R}$. Bourrigaud nous renvoie à cette ultime phase bocagère contemporaine. Illustrant les modalités qui président à la construction du bocage actuel, à une époque très récente, l'auteur récapitule les stades de mise en place du système agropaysager, en réponse à une recherche de productivité nouvelle, notamment au sujet de l'élevage, à laquelle les anciennes landes et incultes n'ont guère résisté.

Le thème 2 , « Des structures et des fonctions évolutives », est dédié aux phases ainsi qu'au rythme d'évolution du réseau bocager. Celles-ci apparaissent comme fortement contrastées, selon les lieux et les époques (F. Di Pietro et C. Pinoteau pour la Gâtine lochoise, p. 147-153; S. Calvo Iglesias et al. pour le nord de la Galice, p. 155-162; L. Le Du-Blayo pour la Bretagne, p. 163-174; S. Vanpenne-Bruhier et al. pour la plaine isérane de Bourg-d'Oisans, p. 175-184). Remontant aux sources des bocages de l'Ouest français, à l'issue d'une implantation et densification progressives allant des $\mathrm{XIV}^{\mathrm{e}}-\mathrm{XV}^{\mathrm{e}}$ jusqu'au milieu du $\mathrm{xx}^{\mathrm{e}}$ siècle, A. Antoine s'interroge sur la question des motivations qui ont commandé la construction bocagère (p. 185-200). Elle récapitule ainsi par le fil de l'histoire l'évolution d'un paysage ouvert attesté au $\mathrm{X}^{\mathrm{e}}-\mathrm{Xv}^{e}$ siècles, au sein duquel alternent aires de cultures et saltus plus ou moins dense, vers un paysage bocager au sens propre, emblématique de l'époque moderne. À sa suite, le lecteur constate au XVI ${ }^{\mathrm{e}}$ siècle un phénomène nouveau, aux lourdes implications socio-économiques, à savoir la diffusion croissante du droit de clore qui, à partir de cette époque, s'applique à ces anciens espaces soumis aux usages collectifs et coutumiers. Or, la principale justification de cette enclosure du paysage réside dans la nécessité déjà ancienne, mais de plus en plus pressante à partir de la fin du Moyen Âge, d'interdire au bétail l'accès aux champs cultivés. Le processus d'enclosure bocagère, répondant à l'essor de l'élevage bovin dans l'Ouest français, est parachevé au XVIII ${ }^{\mathrm{e}}$-début du XIX ${ }^{\mathrm{e}}$ siècle par l'érection d'un réseau de haies par le biais duquel se dessine pleinement la structure du bocage actuel. A. Antoine nous rappelle cependant que ce bocage moderne est encore un espace " poreux ", partagé en métairies et closeries, deux formes d'exploitation plus ou moins compartimentées et de superficie hiérarchisée dont la structure est caractéristique des pays de l'Ouest à la fin de l'Ancien Régime. On y dénombre encore au XviII ${ }^{\mathrm{e}}$ siècle d'importantes surfaces cultivées, le cycle des productions agricoles permettant de dégager tout au long de l'année, en sus des landes, marais et autres friches, de tout aussi considérables parcours de dépaissance pour un cheptel bovin conduit en semi liberté à l'intérieur des limites imposées par les haies autour des cultures. Cette porosité s'estompe par la suite, dans le cours du XIx ${ }^{e}$ siècle, pour aboutir au cloisonnement parcellaire que révèlent les trames bocagères contemporaines.

E. Garnier apporte un regard complémentaire sur les fonctions du bocage moderne, décelant à travers les sources bas-normandes un rôle de protection des cultures contre les aléas climatiques (dont il nous brosse un historique circonstancié [p. 201-211]). Notons que ces considérations concernent aussi bien d'actuels pays bocagers que d'anciens, à l'instar de la Plaine de Caen, commués en openfields au $\mathrm{XIX}^{\mathrm{e}}$ siècle. Ce rôle protecteur se traduit par un dispositif de haies " en rideau ", adapté à la topographie des versants ainsi qu'aux dynamiques d'érosion des sols et de ruissellement au niveau des pentes. Le système haie-talus-fossé apparaît ainsi comme un outil complexe et maîtrisé dans la gestion du drainage ou la protection contre les coups de vent, particulièrement nécessaire à l'arboriculture fruitière. E. Garnier décèle dans l'accomplissement de ce bocage utile l'un des arguments retenus par Arthur Young en faveur des enclosures, qu'il situe à l'origine de la connotation positive apposée au système bocager par l'historiographie française jusqu'aux Caractères de Marc Bloch.

Portant la focale à l'échelle de l'arbre, V. Bernard, F. Épaud et $\mathrm{Y}$. Le Digol se penchent à leur tour sur la question des origines, à travers le prisme de l'étude dendro-archéologique des bois d'œuvre (p. 213-230). Ils jettent un éclairage sur les témoins attestant des pratiques d'émondage dès les temps carolingiens, de même que l'emploi de bois d'œuvre émondés, notamment pour les moulins hydrauliques, et en déduisent que des haies de chêne sont déjà sans doute présentes en nombre dans certains secteurs de l'Ouest, préfigurant l'émergence du bocage à la fin du Moyen Âge. À compter du $\mathrm{Xv}^{\mathrm{e}}$ siècle, la physionomie des bois trahit une sélection permise par un peuplement artificiel planté, ce qui rejoint l'apport des données palynologiques bretonnes. Particulièrement adaptés à certaines contraintes de construction, les bois issus d'arbres émondés deviennent monnaie courante dans l'architecture bretonne dès les alentours de 1450 et durant tout l'Ancien Régime, tandis que les besoins en bois combustible, notamment pour les fours à pain, confèrent une importance première aux réserves de bois et taillis. Ces éléments sont à rapprocher des données naturalistes élaborées par A. Butet $e t$ al. au sujet de la biodiversité des bocages de l'Ouest (p. 231238). Les recensements effectués montrent que l'intensification actuelle du bocage se traduit généralement par une réduction de la diversité faunique au profit d'espèces communes généralistes. S'intéressant au Champsaur alpin, les travaux de P. Campagne et al. portent quant à eux sur la flore ligneuse (p. 239-244). Ils montrent que la structure et l'entretien de la haie ont un impact notable sur la richesse et la composition de celle-ci, qui se manifeste à différentes 
échelles spatiales. Ces observations naturalistes participent pleinement de l'élaboration des actuelles politiques publiques de re-bocagement, notamment en Bretagne, où des systèmes de talus sont mis en place afin de retarder le transfert des écoulements de surface et des polluants agricoles de la parcelle vers les cours d'eau réceptacles. Travaillant à partir d'un SIG mis en œuvre sur trois petits bassins-versants bretons, E. Bocher et al. confirment l'importance des éléments linéaires, bocager mais aussi viaire, dans la dynamique des écoulements de surface (p. 245-260).

D. Pichot ouvre le thème 3, "Les sociétés bocagères ", avec une étude dédiée aux relations unissant paysage et société féodale dans l'ouest de la France, son approche visant à « retrouver les traces de la société médiévale dans le paysage qu'elle a contribué à façonner » à partir d'un rapprochement de données écrites, figurées et palynologiques (p. 263-275). D'emblée, l'historien met en garde contre une conception "téléologique " du paysage qui conduirait à envisager le bocage actuel comme le produit achevé d'une évolution continue, amorcée depuis un mythique pré-bocage attribuable aux XI ${ }^{e}$-XIII ${ }^{e}$ siècles. S'intéressant tout d'abord à la géométrie parcellaire, D. Pichot constate la rareté des ensembles planifiés dans les campagnes de l'Ouest, au profit de nombreux terroirs de superficie réduite qui se déploient sous une forme tantôt circulaire ou ellipsoïdale, tantôt perpendiculaire ou en arête de poisson, clairement datés des $\mathrm{XII}^{\mathrm{e}}$-XIII ${ }^{\mathrm{e}}$ siècles et liés à une phase de mise en culture nouvelle de terres conquises ou reconquises après un hiatus de plusieurs siècles. L'époque médiévale a ainsi imprimé sa marque sur un paysage largement hérité, en l'espèce de petits finages organisés tant à l'échelle individuelle que collective autour de pôles plus ou moins nombreux et décentrés tels que le château, le bourg, l'église et le cimetière, le prieuré. Les cas de configuration parcellaire radioconcentrique étant relativement fréquents autour de ces pôles, le processus d'adaptation des finages médiévaux au paysage préexistant aboutit fréquemment à des constructions hybrides de type radioquadrillé. Au-delà du parcellaire, $\mathrm{D}$. Pichot aborde la question du paysage proprement dit en se fondant sur l'enseignement des sources écrites, et nous dépeint des espaces largement ouverts au sein desquels se côtoient " campagnes " cultivées non encloses et réserves boisées uniformément exploitées. Quoiqu'il existe assurément déjà des haies talutées dans certains secteurs au moins dès le $\mathrm{Ix}^{\mathrm{e}}$ siècle (cartulaire de Redon), fossés et talus restent objectivement rares tandis que prévalent d'autres modes de délimitation des parcelles comme le bornage ou le repérage des confronts. À partir d'une lecture attentive des sources écrites, il semble cependant que ces éléments clés du bocage connaissent une augmentation notable à partir du XIII siècle, en rapport avec des organisations parcellaires elliptiques circonscrites par un talus qui se rencontrent en densité croissante aux abords des villages du second Moyen
Âge. D. Pichot nous montre ensuite comment l'action des sociétés médiévales fut selon lui à l'origine de ces évolutions paysagères. L'accent est mis sur plusieurs facteurs dynamiques inhérents au fonctionnement de la société féodale, à savoir le contrôle et l'encadrement exercés par les élites dans le cadre de la seigneurie et de la paroisse. Les fondations monastiques semblent avoir opéré un rôle notable dans ce processus, dès le $\mathrm{XII}^{\mathrm{e}}$ siècle, tandis que l'aristocratie laïque adopte un comportement sensiblement "parasitaire ", fondé sur le prélèvement seigneurial. Les fondations de bourgs ruraux et prieurés apparaissent comme un facteur de premier plan dans l'essor qui marque cette époque tandis que de nouvelles organisations parcellaires se déploient, dotés d'appareils d'exploitation caractéristiques, métairies et granges, manoirs et prieurés. Une tendance au regroupement des hommes se dessine également dans le cadre affirmé de la paroisse. Dès le haut Moyen Âge et de façon plus rapide, semble-t-il, à compter du XI ${ }^{e}$ siècle, la population tend à se concentrer en villages ou hameaux d'importance variable qui peuvent disputer au bourg son importance. Ce processus est à l'origine de la fixation d'un habitat dispersé (le "village éclaté ") en fonction duquel s'élabore le paysage médiéval, irrigué par de nouveaux réseaux viaires et subdivisé en de multiples finages dont la base identitaire est le centre ecclésial. La mise en valeur de ces finages médiévaux repose sur d'importantes pratiques communautaires touchant notamment des surfaces collectives vouées à un élevage multiforme dont l'importance économique se doit d'être réévaluée. La diffusion de la haie, qui s'accélère à la fin du Moyen Âge, coïncide avec l'effacement progressif de ces usages collectifs au profit d'un nouvel individualisme.

En aucun cas le processus de mutation des paysages au cours du Moyen Âge ne peut donc apparaitre comme un acte fondateur, mais plutôt comme une étape majeure au sein d'une histoire longue et complexe qui est aussi celle des sociétés. Diverses études conduites en dehors du territoire français débouchent sur des résultats convergents. C’est le cas au Québec (N. Gamache et al., p. 277-297), dans la Gascogne du XviıI siècle (R. Sourp, p. 299-307) ou dans le bocage rennais à la même époque (J. Cucarull et P. Bardel, p. 307-322), où l'on constate que l'évolution du paysage bocager est directement induite par les nécessités des sociétés. En rapport avec les préoccupations écologiques $\mathrm{du} \mathrm{XXI}{ }^{\mathrm{e}}$ siècle, diverses études portent également sur la place du bocage dans les paysages et politiques de gestion actuels. Or, les certitudes font parfois défaut, comme le montre l'étude consacrée par M. Cohen et al. à deux petits bassins-versants débouchant sur la baie du Mont SaintMichel (p. 323-330). La sphère juridique se fait également le reflet de ces contradictions très actuelles entre bocages passé et présent, vécu et idéal (P.-Y. Legal, p. 331-343). Dans quelle mesure par ailleurs, les sociétés bocagères affichent- 
elles des traits spécifiques; en d'autres termes, y a-t-il une objective identité des sociétés bocagères? C'est la question que pose J.-M. Constant en opérant la comparaison des paysans modernes de l'Ouest avec ceux de la Beauce et en mettant l'accent sur des coutumes successorales très distinctes davantage que sur les spécificités du paysage (p. 345357). Ceci n'exclut pas pour autant que le bocage n'induit aucune pratique spécifique parmi ceux qui le peuplent, bien au contraire. L. Gourmelen démontre par exemple combien les travaux particuliers des gens du bocage breton occupent une place centrale dans l'organisation sociale et la hiérarchie des valeurs de cette société (p. 359-370). D'autres visions ethnographiques du bocage nous sont proposées par J.Y. Durand au sujet du Minho portugais, terre de la vigne où le bocage, intermittent, est souvent « invisible » (p. 371384), et S. Kergreis et C. Thenail pour l'Armorique et ses bordures de champs (p. 385-395).

Le bocage est encore un espace perçu, générateur d'idées auxquelles est dédié le thème 4 " L'évolution des idées sur le bocage ". Le discours laudatif qui l'emporte aujourd'hui à propos de ce type paysager prend le contre-pied de la critique d'hier motivée par les nécessités reconnues de la modernisation agricole, et tout spécialement du remembrement depuis les années 1960 (S. Périchon, p. 399-410). Le politique n'est pas en reste dans cette sphère des perceptions; c'est ce qu'illustre l'imagerie du bocage chouan élaborée au cours du XIX ${ }^{\mathrm{e}}$ siècle, toujours prégnante aujourd'hui dans ces régions de l'Ouest «bleu » (A. Rolland, p. 411-418). Si l'on se focalise sur l'actualité, le tableau des représentations s'avère étonnamment nuancé. Les facteurs exogènes y jouent bien entendu un rôle actif, notamment les primes et diverses aides à l'agriculture versées à l'intention des exploitants du bocage breton au profit du maintien d'un paysage idéal ( $S$. Périchon). Dans les faits, l'alchimie se révèle complexe. Ainsi, les agriculteurs de Quemper-Guézennec se montrent-ils favorables à l'arasement des talus alors même qu'ils avouent leur attachement, mais à titre d'habitants, aux arbres et haies qui dessinent les lignes de force d'un environnement considéré comme "naturel " et harmonieux (Le Vaillant et al., p. 419-428). C'est que, depuis la fin du $\mathrm{Xx}^{\mathrm{e}}$ siècle, la question du bocage ne concerne plus seulement les agriculteurs; elle mobilise notamment les passions et la soif de nature des "gens de la ville ». Mue par un imaginaire vivace, l'esthétique du bocage et des trognes émondées occupe désormais un champ de plus en plus considérable dans les débats suscités par la gestion des paysages contemporains (M. Toublanc, Y. Luginbhül, p. 429-444). Ce « néo-bocage » des citadins en visite parmi les ruraux est une construction idéale, en rupture avec le bocage traditionnel des agriculteurs. Aussi apparait-il aux yeux de certains comme une forme dénaturée de " béton vert » dont l'unique vocation serait d'embellir les lotissements périurbains. Néanmoins, les citadins angevins ou ceux du Berry de George Sand savent également s'approprier «bouchures » et anciennes haies de frênes têtards, qu'à tort ou à raison ils parent, non sans un certain passéisme nostalgique, d'une infinité de vertus fonctionnelles et écologiques (D. Montambault, p. 445-460; M. Dumont, p. 461-472). Subventions, préconisations, politiques « de pays " alimentent le moteur de cette fabrique contemporaine du néo-bocage, que d'aucuns définiront comme une " fiction paysagère " plus ou moins chargée de sens, à moins qu'il ne s'agisse, peut-être, d'une nouvelle facette de l'entité bocage.

Du passé à l'actuel, de l'agriculteur pragmatique au citadin enthousiaste, de l'arbre émondé aux masses parcellaires, ce copieux ouvrage s'impose comme une véritable somme interdisciplinaire dédiée au bocage dans tous ses états, et remplit sans détour les objectifs annoncés. La masse considérable d'informations qu'il nous livre est d'autant plus précieuse qu'elle est précédée par de savants retours critiques sur l'historiographie des bocages à l'échelle internationale. On pourra regretter, à ce propos, l'absence d'une bibliographie générale récapitulative qui aurait été appréciable tant la thématique et les sources utilisées s'interpénètrent - ce qui, du reste, constitue à nos yeux la plus remarquable réussite de cet ouvrage. Il reste que la lecture de ces actes est parfois ardue en raison d'un chapitrage thématique résolument transversal et, nous a-t-il semblé, quelque peu labyrinthique. Malgré ces quelques réserves, le lecteur trouvera dans ces quelque cinq cents pages une foule de données actualisées qui rendent compte avec vigueur du nouveau visage arboré par les études paysagères, en particulier celles de la nouvelle "école de Rennes». Les processus de transformation à l'œuvre au sein de ces bocages européens et plus lointains sont désormais reconsidérés à la lumière des sciences de l'homme, de la terre et de la nature. Leur apport respectif autorise à discerner les facteurs de durée, de résilience, de mobilité paysagère, de même que les implications emboîtées des héritages techniques et culturels qui sont au cœur et à la source des mutations paysagères. Ces mutations peuvent désormais être considérées dans un espace-temps qu'un recoupement assidu et novateur des sources archéologiques, historiques et paléoenvironnementales est parvenu à redéfinir. Au mythe fixiste des origines et du bocage achevé se sont ainsi substitués les concepts mobiles d'avant- et d'après- ou néo-bocages, et l'approche structuraliste des composantes géographiques, notamment parcellaire, du paysage bocager cède le pas désormais à une lecture davantage fonctionnelle et inscrite dans le temps historique des interactions paysage/ société. On retient notamment le regain fondamental, tant chez les historiens que chez les archéologues ou archéogéographes, du concept de mobilité au sein du temps long de 
l'histoire bocagère, aux dépens de celui, jadis dominant, de continuité-résilience. Le " monde immobile » des campagnes modernes se trouve ainsi repensé à l'aune de l'interdiscipline, de sorte que les évolutions paysagères complexes qui se dessinent aujourd'hui paraissent bien désormais indissociables de celles qui affectent les sociétés humaines, dont le comportement est une réponse et une adaptation à des nécessités changeantes qu'il convient d'éclaircir aussi bien pour les époques les plus reculées que dans l'actualité de ce $\mathrm{XXI}^{\mathrm{e}}$ siècle " écolophile ". En outre, le regard porté à travers ces actes sur l'histoire des perceptions révèle de puissants filtres subjectifs à l'œuvre dans les coulisses du bocage. La dimension affective, psychologique voire psychanalytique des postures et comportements sociaux (relation à la « trogne » de l'arbre émondé...) vient compliquer et accessoirement sans doute embellir l'arrière-plan techno-économique déterminé par les sociétés dites traditionnelles. Depuis des millénaires, l'homme du bocage, conscient ou non de son appartenance identitaire à un univers de la haie qui se manifeste à bien des égards aujourd'hui comme un idéal quelque peu factice, est tout autant un acteur de son environnement qu'une composante à part entière de la diversité paysagère. Célébrées avec brio par ces actes, ces épousailles entre bocages et sociétés sont donc encore loin d'avoir porté tous leurs fruits.

Vincent CARPENTIER

(Inrap/Centre Michel de Boüard-CRAHAM, FRE 3119)

Chancerel, A., Marcigny, C. et Ghesquière, E. (dir.), 2005 - Le plateau de Mondeville (Calvados) : du Néolithique à l'âge du Bronze, Paris, éditions de la Maison des Sciences de l'Homme ("dAf», 99), 208 p.

Le plateau de Mondeville, situé dans la plaine de Caen, a fait l'objet de multiples opérations d'aménagement, précédées de diagnostics et de fouilles préventives entre 1990 et 1995. Trois zones industrielles y ont été implantées sur une emprise géographique relativement limitée : la ZAC du Marché d'Intérêt régional (MIR), la ZAC de l'Étoile et la ZI de Mondeville-sud. Une dizaine de responsables d'opérations se sont succédé sur les différents chantiers archéologiques au cours du temps. Par la suite, la coordination scientifique mise en place en vue d'une publication des sites fouillés n'a été rassemblée que très tardivement. Ces deux facteurs augurent à eux seuls des énormes difficultés rencontrées pour le travail de récolement des données. Cyril Marcigny et Emmanuel Ghesquière ont réalisé un véritable tour de force en rassemblant et harmonisant une documentation inégale, parfois fort lacunaire, en vue de la publication des résultats issus des différentes opérations préventives effectuées. Bien que publié en 2006, l'ouvrage a été rédigé entre 1994 et 1999 (Cf. avertissement, p. 12). Une bibliographie actualisée permet d'estomper les décalages dus à l'avancée de la recherche entre la date de fin de rédaction et celle de la publication. On ne peut que féliciter les auteurs pour l'étendue du travail accompli et souligner la qualité formelle toujours aussi remarquable des publications de la collection dAf.

L'ouvrage s'ouvre sur un chapitre introductif (chap. 1 : Présentation générale des sites), à la suite duquel les différents sites archéologiques sont présentés les uns après les autres dans des chapitres successifs respectant la chronologie, de la phase néolithique VSG à la transition Bronze/Fer. Les chapitres sont de longueur et d'importance variables, en fonction du potentiel archéologique offert par chaque structure ou ensemble fouillé. Chaque opération donne lieu à une présentation détaillée des données collectées sur le terrain. Les mobiliers sont étudiés de façon méthodique et sont très bien documentés. Le texte, toujours fluide, est soutenu en permanence par des illustrations variées et de grande qualité, ainsi que par de nombreux tableaux et graphiques. L'étude du mobilier lithique, par exemple, est systématiquement synthétisée sous forme de tableaux à l'intérieur desquels apparaît la totalité des pièces mises au jour. En bref, les données rassemblées lors des différentes opérations de fouilles sont offertes très généreusement aux lecteurs, donnant une impression de grande transparence dans la transmission des informations, ce qui n'a probablement pas toujours été simple.

Le Néolithique est évoqué à travers trois occupations différentes : une maison danubienne située au Haut-SaintMartin, dans la ZI sud (chap. 2), une série de fosses-silos et une fosse d'extraction datées du Néolithique moyen II, également dans la ZI sud (chap. 3) et une fosse à dépôts d'animaux datés du Néolithique récent dans la ZAC de l'Étoile (chap. 4). Pour cette période, les découvertes du plateau de Mondeville offrent un apport très positif et permettent de compléter les données régionales sur le courant VSG récent et sur le Néolithique moyen II.

La maison danubienne du Haut-Saint-Martin apparaît isolée, contrairement aux villages danubiens "classiques" qui présentent généralement des rangées de bâtiments très rapprochés. Les recherches de maisons mitoyennes n'ont donné aucun résultat. La maison et les fosses d'accompagnement sont bien datées, en particulier par l'outillage lithique en silex, qui place le site à la fin de la période VSG. Ce site complète les données sur la phase du VSG récent et confirme à nouveau la présence du modèle danubien dans l'ouest de la France. Les auteurs supposent d'ailleurs que l'extension 